le portiQue Le Portique

le portiQue Revue de philosophie et de sciences humaines

$22 \mid 2009$

Catastrophe(s)?

\title{
Ouvertures à un concept : la catastrophe
}

\section{Christian Godin}

\section{(2) OpenEdition \\ Journals}

Édition électronique

URL : http://journals.openedition.org/leportique/1993

DOI : $10.4000 /$ leportique. 1993

ISSN : $1777-5280$

\section{Éditeur}

Association "Les Amis du Portique"

Édition imprimée

Date de publication : 10 avril 2009

ISSN : 1283-8594

\section{Référence électronique}

Christian Godin, «Ouvertures à un concept : la catastrophe », Le Portique [En ligne], 22 | 2009, mis en ligne le 10 novembre 2010, consulté le 25 mars 2021. URL : http://journals.openedition.org/leportique/ 1993 ; DOI : https://doi.org/10.4000/leportique.1993

Ce document a été généré automatiquement le 25 mars 2021.

Tous droits réservés 


\title{
Ouvertures à un concept : la catastrophe
}

\author{
Christian Godin
}

1 Les premières visions d'apocalypse ont été religieuses. On peut parler ici d'une véritable constante dans l'imaginaire humain : toutes les sociétés ont cru à la réalité de catastrophes passées ou à venir. Mais ces cataclysmes qu'évoquent les mythologies étaient rarement complets, et lorsqu'ils l'étaient, ils se trouvaient justifiés comme des moyens de régénération. Lors même que la téléologie judéo-chrétienne substituera sa vision linéaire, orientée, du cours des choses, à l'image du cycle, caractéristique des religions antiques, cette idée de régénération ne disparaîtra pas : de fait, c'est bien une humanité nouvelle qui doit surgir après les calamités annoncées dans l'Apocalypse par saint Jean.

Le rationalisme de l'âge classique a rejeté ce mélodrame cosmique et divin au profit de l'idée d'une progression accomplie par l'homme lui-même. À la fin du XVIII siècle, Kant pourra écrire que l'idée d'une fin de tout ne tire pas son origine de la réflexion sur le cours physique des choses de ce monde. Or cela, précisément, n'est plus vrai aujourd'hui. Ce n'est pas, dit Peter Sloterdijk dans une formule reprise de Sartre, ni notre faute ni notre mérite si nous vivons à une époque dans laquelle l'apocalypse de l'homme est quelque chose de quotidien ${ }^{1}$.

Avant même que les guerres mondiales, les totalitarismes et les génocides ne ravagent la scène du monde, l'art et la musique modernes ${ }^{2}$ avaient suggéré ce terrible message : désormais, l'homme vit sous le signe de la catastrophe. Et cela advint au moment même où il commençait à ne plus croire à l'apocalypse ${ }^{3}$. La pensée d'apocalypse n'aura donc été suspendue que pendant un laps de temps fort court, entre Kant et Hiroshima. Symptomatique de ce tournant est le changement de lumière qui baigne la sciencefiction : la légende noire a remplacé la légende dorée.

La catastrophe peut être définie comme un événement d'une intensité tragique maximale accompagné ou suivi de destructions multiples. Elle fait planer sur l'existence humaine une mort de masse. Elle est un événement monstrueux, l'absolu du 
risque et de l'accident. Elle n'est pas seulement un accident d'une puissance particulière ; son énormité dépasse la catégorie de l'accidentel.

5 En littérature, et spécialement dans la tragédie classique, la catastrophe est l'événement décisif qui introduit au dénouement d'une tragédie - d'où l'identification entre catastrophe et tragédie. "Cata » en grec signifie en dessous, au fond, en arrière. Le préfixe désigne le mouvement qui descend, creuse et retourne. Katastrophê veut dire renversement, la strophê renvoyant à l'action de tourner. Dans le cadre d'une conception cyclique du temps, la catastrophe est volontiers pensée comme un retour au point de départ ${ }^{4}$. Dans le contexte d'une conception linéaire du temps, en revanche, qui est la conception moderne, la catastrophe est marquée à la fois par son irréductible singularité et par son irréversibilité. Elle donne l'apparence d'une incommensurabilité entre l'effet (énorme, littéralement) et la cause souvent indétectable, en tout cas imprévisible ${ }^{5}$ - d'où le défi qu'elle représente pour la pensée.

6 La catastrophe est ce qui retourne, dans les trois valeurs du verbe. La catastrophe revient (la strophe y fait renvoi ${ }^{6}$ ) ; la catastrophe bouleverse (les victimes en sont toutes retournées) ; la catastrophe met sens dessus dessous : la surface est enfouie, et le fond mis au jour.

7 Y a-t-il des catastrophes objectives ou bien n'avons-nous affaire qu'à une notion anthropocentrique liée à notre malheur extrême? La catastrophe comme radicale discontinuité est-elle une exception ou bien entre-t-elle dans le cadre de régularités repérables? Ces deux questions engagent les deux lignées sémantiques principales: celle du désastre (humain) et celle de la rupture (objective).

$8 \quad$ Mais s'il est nécessaire de ne pas confondre le concept avec ses usages emphatiques ou métaphoriques ${ }^{7}$, la montée à l'absolu bute sur une aporie. «La seule catastrophe qui paraît claire à tous serait la catastrophe à laquelle personne ne survit ", écrit Sloterdijk ${ }^{8}$. La catastrophe par excellence, celle qui nous permettrait de penser le concept dans toute sa force est par définition celle que nous ne connaitrons jamais. Toutes les catastrophes passées, en effet, n'ont été que des catastrophes partielles et éphémères, par rapport à l'ensemble de l'humanité. En ce sens, elles n'ont pas été de "véritables " catastrophes ${ }^{9}$. Le pire en effet est impossible à penser car la pensée présuppose un monde, fût-il mauvais.

9 Les mythologies sont catastrophistes, car les origines sont catastrophiques autant que les fins. Par opposition, le logos tendra à privilégier la régularité des phénomènes et des lois.

10 Or, à la fin du XVIII ${ }^{\mathrm{e}}$ siècle et au début du XIX ${ }^{\mathrm{e}}$ siècle, des controverses ont agité les sciences de la nature et elles ont porté principalement sur la question de savoir si ce sont les continuités ou les ruptures qui ont ponctué l'histoire de la Terre. Cuvier défendait un point de vue catastrophiste, que ses adversaires ne manqueront pas de dénoncer comme trop soumis au paradigme biblique du Déluge et de l'Apocalypse. Selon les "gradualistes", à l'inverse, les extinctions des espèces vivantes ont été progressives et non pas brutales. Un débat parallèle marqua la naissance de la géologie : au catastrophisme des révolutions du globe défendu par Cuvier s'opposait l'actualisme (ou uniformitarisme) de Charles Lyell : les mêmes causes aujourd'hui observables ont dû agir dans les temps primitifs de la Terre. Le vulcanisme soutenu par les catastrophistes mettait en avant les phénomènes d'explosion tandis que le neptunisme 
défendu par les uniformitaristes privilégiait les phénomènes d'érosion: nouvelle bataille entre le feu et l'eau, sortie cette fois de son cadre mythologique.

$11 \mathrm{Au} \mathrm{xx}^{e}$ siècle, le catastrophisme dans les sciences de la Terre sera réactualisé par deux découvertes majeures : en climatologie, celle de la fin brutale de la dernière glaciation, qui provoqua la hausse du niveau des océans de plusieurs dizaines de mètres, et en paléontologie la découverte des astroblèmes. On appelle impactisme la théorie catastrophiste selon laquelle la vie sur Terre et l'existence de la Terre elle-même ont été en grande partie conditionnées par la chute d'astéroïdes sur le globe.

La notion de catastrophe a été à l'époque contemporaine utilisée dans toutes les sciences, de la biologie aux mathématiques. Kurt Goldstein a appelé catastrophe le comportement désordonné en rupture avec l'ordre qui semble régir la conduite (adaptative) de l'individu dans son milieu familier. C'est cet usage qui a conduit René Thom à utiliser le terme pour désigner sa théorie mathématique.

La théorie des catastrophes est une morphologie générale des discontinuités ${ }^{10}$. La catastrophe est un type de discontinuité qu'un système dynamique doit franchir pour passer d'une région de son déploiement dans une autre. La théorie des catastrophes rend raison des structures en justifiant dynamiquement leur apparition et leur stabilité. Son approche morphologique entend résoudre l'antagonisme entre le point de vue réductionniste qui abstrait les constituants élémentaires et le point de vue finaliste qui privilégie la structure fonctionnelle de l'ensemble. Par la fécondité comprise dans sa généralité même, cette théorie a pu connaître des applications diverses dans presque tous les domaines de la recherche scientifique : le mathématicien Christopher Zeeman, qui a travaillé sur le comportement d'agressivité du chien ${ }^{11}$, a proposé des applications en physique et en biologie, mais aussi en économie ${ }^{12}$ et en politique, en sociologie ${ }^{13}$ et en psychologie ${ }^{14}$.

14 Dans l'existence particulière ou collective des hommes, une catastrophe est un événement unique dont le sens est contradictoirement de ne rester justement jamais unique. D'abord parce qu'une catastrophe se répète. Ensuite parce qu'une catastrophe en enclenche une série indéfinie d'autres.

15 La catastrophe semble unique mais elle ne frappe presque jamais d'un coup. Elle tue et dévaste par à-coups successifs ${ }^{15}$. Günther Anders appelait « syndrome de Nagasaki » la répétition " désinvolte, irréfléchie, immotivée » de la première catastrophe ${ }^{16}$.

Dans son Discours sur l'origine et les fondements de l'inégalité parmi les hommes, Rousseau évoque l'origine catastrophique de la propriété privée. Origine doublement catastrophique : l'appropriation est une catastrophe en soi et elle en engendre une longue série d'autres : «Le premier qui ayant enclos un terrain, s'avisa de dire ceci est à moi et trouva des gens assez simples pour le croire, fut le vrai fondateur de la société civile. Que de crimes, de guerres, de meurtres, que de misères et d'horreurs n'eût point épargnés au genre humain celui qui arrachant les pieux ou comblant le fossé, eût crié à ses semblables : Gardez-vous d'écouter cet imposteur; vous êtes perdus si vous oubliez que les fruits sont à tous et que la terre n'est à personne ${ }^{17}$. Mais aussitôt après, Rousseau précise que cet événement a été préparé par beaucoup d'autres antérieurs et donc que la catastrophe s'inscrit elle-même dans une certaine continuité : « Mais il y a grande apparence qu'alors les choses en étaient déjà venues au point de ne plus pouvoir durer comme elles étaient ; car cette idée de propriété, dépendant de beaucoup d'idées antérieures qui n'ont pu naître que successivement, ne se forma pas tout d'un coup dans l'esprit humain : il fallut faire bien des progrès, acquérir bien de l'industrie 
et des lumières, les transmettre et les augmenter d'âge en âge avant que d'arriver à ce dernier terme de l'état de nature ${ }^{18}$.

L'histoire contemporaine ne manque pas d'exemples de ces séries rétrospectives d'événements préparatoires à la catastrophe terminale: la Shoah (qui signifie catastrophe) a eu pour contexte la catastrophe de la Seconde Guerre mondiale, laquelle dérive de la catastrophe représentée par l'arrivée au pouvoir des nazis, qui ont profité de la catastrophe de la Grande Crise de 1929, laquelle n'aurait peut-être pas eu lieu sans la catastrophe de la Première Guerre mondiale, etc. La bombe atomique fut la résultante de ces deux catastrophes absolues en matière politique que sont le totalitarisme et la guerre mondiale, et dont la puissance d'extermination n'a pas eu sa pareille dans toute l'Histoire. En ce sens, la catastrophe est déjà là avant qu'elle n'arrive : on songe à l'attelage tiré par des chevaux fous qui filent vers le précipice. Il y a toujours avant la chute un certain parcours.

L'accident, fait observer Paul Virilio ${ }^{19}$, ne surgit pas de l'extérieur ainsi que le suggère son étymologie (" tomber sur »), il est inhérent à l'invention. Chaque progrès technique génère son type d'accident : le bateau fait le naufrage, le train le déraillement, l'avion le crash, etc. L'accident n'est pas un événement inopiné qui vient du dehors mais une soudaine transformation de la matière dans l'espace.

19 La tragédie tient au contraste pathétique entre la toute-puissance objective du Destin et la fragilité de l'être humain. Il n'y a de catastrophe que pour l'homme et contre lui. Lorsque la comète Kohoutek a percuté la planète Jupiter, le choc a été d'une violence inimaginable, pourtant ce n'était pas une catastrophe.

20 La catastrophe peut être d'origine surnaturelle (on l'appelle alors apocalypse), naturelle ou humaine. Mais c'est toujours l'humanité qui en est la victime - quand bien même elle ne serait pas la seule à l'être (l'Apocalypse détruit le monde entier).

21 Le cataclysme (du grec kataklusmos, inondation) est à l'origine une catastrophe naturelle. Il a fini par s'identifier à n'importe quel bouleversement dramatique et mortifère, même à ceux où la nature n'a aucune part. Il en va de même avec le désastre - qui dans la croyance astrologique renvoie au fait d'être né sous un mauvais astre.

22 L'origine surnaturelle de la catastrophe étant écartée, peut-on ranger dans une catégorie commune les «attentats» du 11 septembre 2001 et le tsunami de 2004, un génocide et un tremblement de terre? L'opposition établie entre les catastrophes naturelles et les catastrophes humaines semble aujourd'hui à la fois élémentaire et indiscutable.

Les catastrophes proprement humaines, c'est-à-dire celles qui ont l'homme pour seule cause directe peuvent être de type politique ou technique. Les violences (dont la guerre et le génocide sont les formes extrêmes) et le despotisme (dont le totalitarisme est la manifestation exacerbée) sont des catastrophes politiques. Les accidents technologiques (comme celui de Tchernobyl) et les désastres écologiques sont des catastrophes techniques. La guerre moderne conjoint ces deux dimensions, politique et technique.

24 Les textes législatifs qui définissent la catastrophe naturelle font état de "l'intensité anormale d'un agent naturel ». Mais l'anormalité des catastrophes, même si elle semble fondée sur des mesures statistiques, reste une valeur anthropocentrée : c'est à l'échelle de la vie humaine ou de l'existence collective des hommes (un souffle comparé aux âges géologiques) que l'on établira que tel phénomène est « normal » ou pas. On peut donc 
se demander si l'expression de catastrophe naturelle n'est pas contradictoire en soi. En effet, toute catastrophe est, in fine, humaine, et même si elle n'a pas une cause humaine elle a un impact humain forcément révélateur de faiblesses humaines.

Toutes les fois qu'un cataclysme frappe un pays, particulièrement du tiers-monde, là où les victimes sont toujours les plus nombreuses, et les plus démunies, nous revient en mémoire la lettre que Rousseau écrivit à Voltaire, il y a deux siècles et demi, en réponse à son Poème sur le désastre de Lisbonne. Le tremblement de terre de Lisbonne (1755) provoqua chez les penseurs du siècle des Lumières une véritable commotion. Nombre d'historiens aujourd'hui considèrent que ce cataclysme sonna le glas de la démarche philosophique que Leibniz avait fait connaître sous le nom de théodicée, qui consiste à justifier le mal au nom d'une harmonie supérieure. Après le désastre de Lisbonne, il semblait désormais impossible de croire en une Providence juste et bonne. Rousseau répond à Voltaire (qui lui avait envoyé son poème) qu'excepté la mort «qui n'est presque un mal que par les préparatifs dont on la fait précéder », « la plupart des maux physiques qui affligent l'homme sont de son fait». "Convenez, écrit-il à propos de la ruine de Lisbonne, que la nature n'avait point rassemblé là 20000 maisons de six à sept étages ", "et que si les habitants de cette grande ville eussent été dispersés plus également, et plus légèrement logés, le dégât eût été beaucoup moindre et peut-être nul ». À Voltaire qui demandait avec une feinte naïveté que les catastrophes eussent lieu dorénavant au milieu des déserts, là où personne n'habite, Rousseau répond justement qu'il ne manque pas d'y avoir des tremblements de terre en ces lieux vides d'hommes ${ }^{20}$.

26 Les tremblements de terre qui frappent tel pays pauvre, comme les cyclones qui régulièrement balaient les îles basses du malheureux Bangladesh, montrent assez combien Rousseau avait raison de considérer qu'il n'y a pas, en fait, de catastrophes naturelles mais seulement des catastrophes humaines. Chassés de leurs terres par la misère, les Bangladeshis construisent des abris de fortune dans les endroits les plus exposés - aussi la nature comme un mauvais destin semble-t-elle s'acharner sur eux. Mais c'est de l'injustice des hommes que ces gens sont d'abord victimes; seulement nous trouvons encore dans la nature, comme au XVIII siècle les philosophes trouvaient dans la Providence, un alibi idéal pour n'avoir pas à nous interroger sur la façon dont la société fait mal vivre bien des hommes.

Les menaces qui mettent en péril aujourd'hui la Biosphère confirment, et au-delà, les diagnostics les plus pessimistes. La paléontologie nous a révélé que l'histoire de la Terre a été ponctuée par des extinctions de masse qui, par cinq fois, ont détruit $95 \%$ des espèces vivantes ${ }^{21}$. L'hypothèse la plus communément admise aujourd'hui attribue ces catastrophes à la collision d'un astéroïde avec notre planète. Désormais les spécialistes parlent d'une sixième extinction pour désigner celle qui se déroule sous nos yeux ${ }^{22} . \mathrm{Si}$ l'on excepte le choc d'un astéroïde (dont l'éventualité ne peut être exclue), aucune catastrophe désormais, même celle qui affecterait la totalité de l'écosystème terrestre, ne peut être dite naturelle. Les catastrophes sont humaines, trop humaines.

«En fait, interroge Kant, pourquoi les hommes s'attendent-ils au juste à une fin du monde ? Et, celle-ci étant admise, pourquoi précisément à une fin dans la terreur (pour la plus grande partie de l'espèce humaine) ? ${ }^{23}$. La première réponse que Kant donne à cette question tient dans l'espèce de décalage qui existe entre l'homme et le monde : "Le monde ne mérite de durer que dans la mesure où les êtres raisonnables qui le peuplent sont conformes au but final de leur existence; dès l'instant que ce but risque 
de ne pas être atteint, la création elle-même leur paraît sans objet, comme une pièce de théâtre dépourvue de tout dénouement et qui ne permet pas de reconnaître une intention rationnelle $"{ }^{24}$. L'idée d'une fin du monde reposerait par conséquent sur l'absence d'une foi en une finalité purement humaine de l'histoire, sur l'absence de croyance au progrès. Inversement, l'idée de progrès, à partir du moment où elle s'imposera, à l'époque de Kant justement, refoulera dans la superstition les pensées d'apocalypse. Et lorsque cette idée sera ébranlée par les tragédies sans nom de l'histoire contemporaine, de nouveau la crainte de l'apocalypse resurgira.

La seconde réponse que Kant donne à la question de savoir pourquoi les hommes s'attendent à une fin du monde se fonde sur l'idée de la corruption de la nature humaine. Et nous retrouvons ici un même mouvement en trois phases : c'est parce que l'homme n'avait pas tout lieu d'être fier de lui qu'il s'est d'abord attendu à une destruction de son être; lorsqu'il se mit à la place de Dieu et crut à son infinie perfectibilité, alors l'apocalypse disparut de son horizon, la téléologie de l'histoire remplaçant la théologie; enfin, sous le coup des catastrophes par lui-même provoquées, il se remit à aspirer, comme le Wotan de Wagner, à sa propre fin. Les apocalypses religieuses ont toutes pour fondement un jugement moral sur le caractère corrompu du monde. Mais la croyance dans le renouveau rend impensable la destruction totale et définitive de l'humanité.

On parle d'eschatologie universelle (par opposition à l'eschatologie individuelle qui ne concerne que l'homme singulier) pour désigner le récit concernant la fin de l'univers tout entier. L'eschatologie universelle résulte ou bien d'un destin inéluctable ou bien d'une décision prise par une puissance divine. Comme l'a montré Mircea Eliade, tous les mythes du déluge sont également des mythes de la régénération. Si l'humanité est exterminée, elle ne l'est pas entièrement (un couple survit) et de ce reste infime une autre humanité provient. L'idée de rédemption est à la fois un moyen d'expliquer, de justifier et de conjurer la catastrophe. Mais elle implique le caractère à la fois partiel et relatif de celle-ci. Aujourd'hui, nous ne croyons plus à la rédemption. La survie nous suffit. C'est à partir de cet horizon de survie que Günther Anders parlait d'une apocalypse nue, c'est-à-dire d'une apocalypse qui ne serait la promesse d'aucun Royaume ultérieur ${ }^{25}$.

31 La catastrophe est ce qui met en échec le désir de toute-puissance et le rend dérisoire. Ainsi peut-on comprendre la prolifération de son imaginaire au cinéma et en littérature. Mais le spectacle de la catastrophe n'entre pas en contradiction avec le volontarisme optimiste du capitalisme prométhéen, il en est plutôt l'envers ${ }^{26}$.

$\mathrm{Au}$ sens courant, le catastrophisme est la propension à considérer la catastrophe comme le mode normal d'apparition du réel. Il peut aller, ainsi qu'on le voit dans les médias contemporains, jusqu'au culte de l'accident. Cette connivence du catastrophique et du spectaculaire n'est pas récente car si le sens théâtral de la catastrophe n'est pas premier, il a joué aux $\mathrm{XVI}^{\mathrm{e}}$ et $\mathrm{XVII}$ e siècles le rôle de paradigme pour les accidents les plus radicaux de la vie (maladies, fléaux) tout comme si le monde était une scène. C'est d'autre part le dénouement souvent sanglant des tragédies qui a donné à la catastrophe son sens résolument négatif. Aristote avait établi sa théorie de la catharsis à partir de la tragédie d'Ædipe. Les spectacles de catastrophes ont sans doute une fonction analogue de délivrance et de prophylaxie. De nombreux films et feuilletons télévisés mettent en scène des survivants pour lesquels la catastrophe est déjà passée. L'une des leçons fondamentales de la psychanalyse, rappelle Slavoj Zizek, 
est que « les images de catastrophe, loin de donner accès au Réel, peuvent fonctionner comme un bouclier qui protège du Réel ${ }^{27}$. Les fantasmes et images de catastrophes ont vacciné l'homme moderne contre la seule catastrophe à laquelle il ne survivrait pas.

Comme la violence, et la mort en général, la catastrophe suscite un mélange ambivalent de répulsion et de fascination. Il existe une fascination pour la catastrophe, un désir de catastrophe ${ }^{28}$ sur lesquels le "catastrophisme éclairé » ne cessera de buter ${ }^{29}$. Notre modernité, qui a le culte de l'accident (l'accident est ce qui permet, par le biais de leur mise en images, donc de leur mise en scène, de donner une valeur marchande aux affects) notre modernité fait un usage hyperbolique de la catastrophe ${ }^{30}$. Le déni de la catastrophe est plus répandu que le catastrophisme : il repose sur ce paralogisme qui déduit un « jamais» du " pas encore». Nous ne voulons pas croire ce que nous savons, cela est vrai de notre mort collective comme de notre mort individuelle.

Le « catastrophisme éclairé » qu'entend promouvoir Jean-Pierre Dupuy ${ }^{31}$ repose sur le stratagème mental suivant: si nous croyons la catastrophe évitable, alors elle se produira; mais si nous sommes convaincus qu'elle est certaine, alors nous avons une chance de l'éviter.

Ces termes rappellent ceux, classiques, de la logique modale du possible et $\mathrm{du}$ nécessaire, qui avaient mis aux prises Aristote avec les Mégariques. Les Mégariques soutenaient qu'un événement ne peut être dit possible qu'à partir du moment où il arrive réellement. Autrement dit, c'est la nécessité du réel qui seule peut rétrospectivement constituer le possible en possible. Pour Aristote, à l'inverse, le possible laisse ouverte la bifurcation de l'existence et de l'inexistence : quelque chose peut être en puissance sans être jamais en acte.

Le catastrophisme éclairé traite contradictoirement la catastrophe future sur le mode d'une fatalité ne résultant d'aucune intention humaine mais que nous sommes libres néanmoins d'écarter ${ }^{32}$. Il s'agit de séparer l'humanité de sa propre violence en faisant de celle-ci un destin sans conscience mais capable de nous anéantir : « la ruse consiste à faire comme si nous étions sa victime tout en gardant à l'esprit que nous sommes la cause unique de ce qui nous arrive ${ }^{33}$.

Les catastrophes d'aujourd'hui tendent à devenir invisibles ${ }^{34}$. Et la désarmante accoutumance de la conscience humaine au pire ne rend pas facile la tâche d'avertissement. Évoquant le témoignage de William James, qui était présent lors du grand tremblement de terre de San Francisco de 1906, Bergson a finement décrit l'état d'esprit paradoxal dans lequel se trouvaient les Français avant 1914 face à l'éventualité d'une nouvelle guerre. Celle-ci, dit Bergson, leur apparut à la fois comme probable et comme impossible - jusqu'à se présenter, après avoir éclaté, avec toute sa fausse évidence. Ce caractère "bon enfant» de l'Accident était ce qu'il y avait de plus frappant ${ }^{35}$.

Le catastrophisme total - l'annonce de la complète destruction de l'humanité - est une position irréfutable, car tant que le chaos prévu n'est pas arrivé, il peut toujours être dit possible mais s'il arrive, nul ne sera plus là pour le constater ${ }^{36}$. Il existe aussi une perversité propre au catastrophisme total qui peut faire souhaiter à celui qui y est pris que la catastrophe arrive, d'une part pour la jouissance d'avoir raison, d'autre part pour susciter la réaction appelée de ses vœux. 
39 Mais comment convaincre de la nécessité du pire sans susciter une panique contreproductive ? L'un des plus grands défis est celui de la nécessité de prendre des décisions dures sur fond de connaissances molles.

Günther Anders et Hannah Arendt ont réfléchi sur ce tournant: désormais l'homme n'est plus capable de se représenter ce qu'il fait. De là, un mal inédit, monstrueux, dont Eichmann a été l'incarnation. Ce que nous pouvons faire désormais est plus grand que ce que dont nous pouvons nous faire une image; entre notre capacité de fabrication et notre capacité de représentation, un fossé s'est ouvert, qui va s'élargissant de jour en jour; notre capacité de fabrication est sans bornes, tandis que notre capacité de représentation est limitée ${ }^{37}$. Ce hiatus correspond à celui que Descartes notait entre l'entendement (fini) et la volonté (infinie) - à cette différence toutefois que le pouvoir technique réalisé et espéré a aujourd'hui fait basculer la volonté du côté des désirs et des fantasmes, c'est-à-dire de l'inconscient. "Par son énormité, écrit Anders, c'est-àdire du fait même qu'elle est trop grande pour être seulement mienne ou tienne, la catastrophe nous décharge de notre peur de la même manière [...] que les grandes entreprises et les grandes actions auxquelles nous collaborons avec d'autres, en tant que travailleurs, nous déchargent de notre responsabilité ${ }^{38}$.

41 L'analyse de Günther Anders a pour sens d'arracher la catastrophe à sa singularité. Auschwitz et Hiroshima n'ont pas eu lieu seulement une fois pour toutes; désormais, ils hypothèquent la totalité de notre futur ${ }^{39}$. Notre monde est celui dans lequel d'autres Auschwitz et d'autres Hiroshima sont possibles. Seulement, cette présence, cette actualité perpétuelles sont l'objet d'un refoulement: l'événement catastrophique est "dissimulé au cœur même de notre négligence " ${ }^{40}$. Désormais, depuis Auschwitz et Hiroshima, nous sommes dans « le temps de la fin » et rien ne pourra jamais faire que nous n'y soyons plus. Nous sommes, dit Anders, la première génération des derniers hommes ${ }^{41}$. Même si nous ne vivons pas actuellement la fin des temps, nous sommes dans le temps de la fin, ce qui signifie qu'à tout moment la fin peut advenir. Ce temps de la fin est définitif : nous ne pourrons jamais aller en deçà de lui, revenir à un avant de lui. Pour toujours notre temps est celui de la fin : «il ne peut plus être relayé par un autre temps mais seulement par la fin ${ }^{42}$. Certes, nous pouvons obtenir un moratoire, œuvrer en direction d'une prolongation (forcément relative) de ce temps de la fin " mais en supposant que nous remportions cette victoire, il est certain que ce temps restera ce qu'il est, à savoir un temps de la fin ${ }^{43}$.

La catastrophe rendrait-elle impossible toute stratégie? Günther Anders a analysé le paradoxe de l'intention auto-invalidante de la dissuasion nucléaire: pour que la dissuasion soit réelle, il est nécessaire d'afficher une détermination sans faille à déclencher l'apocalypse afin d'être certain qu'on n'aura pas à le faire. On retrouve ici la structure logique du paradoxe de Jonas ${ }^{44}:$ c'est lorsqu'une catastrophe apparaît comme inéluctable qu'il reste une possibilité de l'éviter. Comme l'écrit François L'Yvonnet à la fin de sa préface pour Le Temps de la fin: " Les philosophes n'ont fait qu'interpréter et transformer le monde. Désormais, il importe de le conserver ${ }^{45}$. 


\section{NOTES}

1. P. SLOTERDIJK, La Domestication de l'être, trad. O. Mannoni, Paris, Mille et Une nuits, 2000, p. 74.

2. Le cubisme et Le Sacre du printemps ont souvent été vus comme les signes précurseurs de la dislocation que les corps allaient subir dans les tranchées de la guerre de 1914-1918.

3. L'apocalypse n'a pas d'abord le sens de catastrophe, mais celui de révélation sacrée sur la fin de toutes choses. Le verbe apokaluptein en grec est la traduction du verbe hébraïque galosh, qui signifie révéler, et plus précisément, révéler des secrets et des mystères. Les terribles menaces qui aujourd'hui pèsent sur le monde ont suscité un retour en force des croyances apocalyptiques : la majorité des Américains croient à la bataille finale d'Armageddon, quant au monde arabo-musulman, il est massivement pris par l'imaginaire apocalyptique.

4. La succession des âges en Grèce ancienne (Hésiode) et en Inde est catastrophique : la fin du dernier âge (âge de fer chez les Grecs, âge de Kali en Inde) débouche sur un renversement qui reconduit à l'âge d'or premier.

5. La terrible marée noire qui frappa les côtes de l'Alaska en 1989 a eu pour cause première l'ivresse d'un marin.

6. Il y a une volte identique dans la révolution, laquelle est une forme de catastrophe.

7. Le langage commun contemporain cultive volontiers l'emphase et les superlatifs au moins autant que les litotes et les diminutifs - aussi le moindre incident domestique, le plus léger contretemps dans une existence frappée par la banalité seront-ils appelés des " catastrophes ». Une réflexion philosophique s'attachera à garder au terme tout son poids de tragique.

8. P. SLOTERDIJK, La Mobilisation infinie. Vers une critique de la cinétique politique, trad. fr., Paris, Christian Bourgois, 2000, p. 108.

9. Les seules catastrophes complètes ont été celles qui ont frappé un petit peuple comme celui des Tasmaniens exterminés jusqu'au dernier par les colons blancs.

10. R. тном, Paraboles et catastrophes, Paris, Flammarion, 1983.

11. Un changement brusque affecte la physiologie de l'animal (museau, oreilles) et son comportement.

12. Avec l'étude des krachs boursiers, en particulier.

13. Analyse des émeutes dans les prisons, du comportement des pirates de l'air...

14. Analyse des maladies maniaco-dépressives.

15. Au premier impact sur l'une des Twin Towers le 11 septembre 2001, a succédé un second impact, puis l'effondrement des deux tours. Ainsi la sidération de la première catastrophe n'a pas eu le temps de se résorber. Le tsunami de 2006 a également frappé par à-coups répétés.

16. L'explosion atomique qui détruisit la ville de Nagasaki le 9 août 1945 a eu lieu trois jours seulement après celle qui réduisit Hiroshima en cendres.

17. J.-J. RousSEAU, Discours sur l'origine et les fondements de l'inégalité parmi les hommes, CEuvres complètes III, Paris, Gallimard, Bibliothèque de la Pléiade, 1964, p. 164.

Orthographe modernisée.

18. Ibid.

19. P. VIRILIO, L'Accident originel, Paris, Galilée, 2005. 
20. Lettre de Jean-Jacques RouSSEAU à M. de Voltaire, 18 août 1756, Euvres complètes IV, Paris, Gallimard, Bibliothèque de la Pléiade, 1969, p. 1061-1062.

21. La cinquième, qui a eu lieu il y a 65 millions d'années, est la plus célèbre : c'est elle qui a provoqué la fin des dinosaures.

22. Voir R. LEAKEY et R. LEWIN, La Sixième Extinction, trad. fr., Paris, Flammarion, 1999.

23. E. KANT, La Fin de toutes choses, trad. H. Wizmann, CEuvres philosophiques III, Paris, Gallimard, Bibliothèque de la Pléiade, 1986, p. 314.

24. Ibid.

25. G. ANDERS, Le Temps de la fin, trad. fr., Paris, L'Herne, 2007, p. 88.

26. Lorsque la mort est esquivée, et que la catastrophe n'atteint que les choses, la situation devient franchement comique : tel est le ressort essentiel du burlesque. C'est dans la période qui s'écoule entre 1930 et 1950, et qui fut la plus catastrophique de la modernité, que triompha le cinéma burlesque américain.

27. S. ZIZEK, Fragile absolu ou Pourquoi l'héritage chrétien vaut-il d'être défendu ?, trad. F. Théron, Paris, Flammarion, 2008, p. 113.

28. Nombre de fictions littéraires, cinématographiques, télévisuelles en témoignent. 29. Il y a bien de l'ivresse chez les concepteurs d'engins de destruction massive. Lorsqu'il inventa la « superbombe » (la bombe $\mathrm{H}$, à fusion thermonucléaire), Edward Teller se justifia par « l'esprit d'aventure » et la conquête d'un « domaine nouveau ». Même Oppenheimer, taraudé par le doute, épouvanté par une possible destruction totale de l'humanité, vit dans ce programme d'armement nucléaire quelque chose de « particulièrement délicieux sur le plan technique ». Un peu plus tard, Ken Alibek, responsable des recherches sur les armes bactériologiques en Union soviétique, décrivait ainsi son état d'esprit : « les résultats de mes travaux pouvaient servir à tuer des gens, mais je ne pouvais pas concevoir comment réconcilier ce savoir avec le plaisir dérivant de la recherche » (voir J.-J. SALOMON, Le Scientifique et le Guerrier, Paris, Belin, 2001).

30. L'excès joué/déjoué ( « c'est la cata ! ») est une stratégie de conjuration. Nommés « catastrophes » les incidents de la vie quotidienne sont une manière indirecte et inconsciente de neutraliser magiquement la catastrophe réelle qui est la mort.

31. Pour un catastrophisme éclairé. Quand l'impossible est certain, Paris, Seuil, 2004 ; Petite métaphysique des tsunamis, Paris, Seuil, 2005.

32. J.-P. DUPUY, Petite métaphysique des tsunamis, op. cit., p. 28.

33. Ibid., p. 100.

34. Jean-Pierre Dupuy parle d'invisibilité du mal à propos de la contamination nucléaire (J.-P. DUPUY, « Tchernobyl et l'invisibilité du mal », Esprit, mars-avril 2008, Le temps des catastrophes, p. 67-78).

35. H. BERGSON, Les Deux Sources de la morale et de la religion, Euvres, Paris, PUF, 1970, p. 1110 .

36. Des ouvrages et des films ont bien tenté d'imaginer la Terre après l'apocalypse. Mais la représenter sans survivants, c'est encore supposer une conscience qui en est le témoin.

37. G. ANDERS, Nous, fils d'Eichmann, trad. S. Cornille et Ph. Ivernel, Paris, Payot et Rivages, 2003, p. 52.

38. G. ANDERS, Le Temps de la fin, op. cit., p. 49.

39. D'où le titre Nous, fils d'Eichmann : « Nous tous [...] sommes également des fils d'Eichmann. Du moins des fils du monde d'Eichmann » (op. cit., p. 104). 
40. G. ANDERS, L'Obsolescence de l'homme, trad. Ch. David, Paris, Éditions Ivrea, 2002, p. 262.

41. G. ANDERS, Le Temps de la fin, op. cit., p. 20.

42. Ibid., p. 116.

43. Ibid., p. 117.

44. Dans le Livre de Jonas, qui fait partie de la Bible, Yahvé demande à son prophète Jonas d'annoncer la destruction imminente de la ville de Ninive, parce que ses habitants vivent dans le péché. Jonas prophétise. Terrifiés, les habitants quittent leur vie de péché. Le temps passe, sans que rien n'arrive. Désorienté, Jonas se retourne contre Yahvé et se plaint d'avoir perdu son crédit de prophète. Yahvé lui répond : Je t'ai demandé de prophétiser la destruction de Ninive pour n'avoir pas à la causer. Les économistes et les sociologues ont étudié des phénomènes analogues de prédiction auto-invalidante dans les sociétés contemporaines : si, par exemple, un embouteillage est annoncé, il a des chances de ne pas arriver précisément parce qu'il a été annoncé. Durant toute l'année 1999, il a été question du « bug de l'an 2000 ». De nombreuses entreprises ont dépensé des grosses sommes d'argent pour éviter la catastrophe informatique annoncée. Le bug de l'an 2000 n'a pas eu lieu. Reste évidemment la possibilité de croire que de toute façon il n'aurait pas eu lieu. Pour que le processus de prédiction auto-invalidante s'enclenche en effet, il faut que l'information soit diffusée partout, considérée comme vraie et surtout débouche sur une décision collective rationnelle : des conditions rarement, voire jamais réunies.

45. G. ANDERS, Le Temps de la fin, op. cit., p. 10.

\section{RÉSUMÉS}

L'idée moderne de catastrophe apparaît à la fois en continuité et en rupture avec l'idée mythologique et religieuse d'apocalypse. La catastrophe est l'absolu du risque et de l'accident. Elle représente l'événement dans ce qu'il a de plus monstrueux. Y a-t-il des catastrophes objectives ou bien avons-nous affaire à une notion anthropocentrique liée à notre malheur extreme? La catastrophe comme radicale discontinuité (tel est son sens formel en mathématiques) est-elle une exception ou bien entre-t-elle dans le cadre de régularités repérables? Ces deux alternatives engagent deux lignées sémantiques principales: celle du désastre humain et celle de la rupture objective. Alors que Voltaire s'était servi du tremblement de terre de Lisbonne pour contester l'ordre providentiel de la nature, Rousseau fut le premier à désigner l'être humain comme le seul responsable (et pas seulement le seul concerné) de la catastrophe. L'augmentation et l'intensification du pouvoir de la technique sur les milieux naturels et sociaux ont largement confirmé cette intuition de Rousseau. Quant au catastrophisme, il peut être contradicttoirement affectif et stratégique, redoublant ainsi ce que la monstruosité du phénomène peut avoir de terrifiant mais aussi de fascinant.

The modern notion of catastrophe appears both to continue the mythological and religious notion of apocalypse and to depart from it. The catastrophe is the absolute form that risk and accident can take. For any given event, disaster is the most monstrous possibility, the ultimate expression of said event. 
Are there objective disasters, or are we dealing here with an anthropocentric notion somehow emanating from our extreme unhappiness? Is the catastrophe seen as radical discontinuity (the formal meaning of the word in mathematical terms) an exception, or is it part of a framework of regular, noticeable occurrences? These two possibilities call to two main semantic paths: the human catastrophe or the objective fracture. Where Voltaire had used the Lisbon earthquake to challenge the idea of a providential order in nature, Rousseau was the first to point to humans as sole responsible for the disaster (and not only as sole group concerned by it). The increase and intensification of the technology's power over natural and social environments have since largely confirmed Rousseau's intuition...

Unter dem Wort Katastrophe versteht man das absolute Risiko, den absoluten Unfall, den ungeheuerlichsten Vorfall. Handelt es sich bei Katastrophen um Objektivität oder haben wir es mit einem anthropozentrischen Begriffe zu tun, der mit unserem extremen Unglück in Zusammenhang gebracht werden muss?

\section{AUTEUR}

\section{CHRISTIAN GODIN}

Christian Godin est né en 1949. Il est professeur agrégé de philosophie, docteur d'état et a enseigné pendant 23 ans en classe de terminale de lycée. Il est maître de conférences de philosophie à l'université Blaise-Pascal de Clermont-ferrand depuis 1999. Auteur d'une trentaine d'ouvrages et d'une centaine d'articles, parmi ses dernières publications : La Guerre, Éditions du temps, 2006 ; Croyance et superstition, Éditions Cécile Defaut, 2007 ; Le Triomphe de la volonté, Champ Vallon, 2007 ; Le Racisme, Éditions du temps, 2008. 